\title{
Sponsors and Investigators in Food Science: Vicious Circle or Virtuous Circle?
}

$\mathrm{T}$ here is large commercial interest in research in nutritional science. The European Union has, therefore, issued a regulation which demands the need to substantiate specific health claims related to dietary products within the European Union (Regulation No. 1924/2006 of the European Parliament and of the Council of December 20, 2006). This has led to the first rejection of specific "health claims" (http://www.efsa. europa.eu/EFSA/ScientificPanels/efsa_locale-1178620753812_NDA. $\mathrm{htm}$ ) through a panel of scientific experts. In nutritional science, in particular, the independency of investigators and the role of manufacturers is therefore a central question. Investigators and manufacturers can form a virtuous circle which improves nutritional support to the population or a vicious circle in which health products are falsely advertised as being beneficial for health. During the next few years, in the face of an expected limitation of financial support, optimization of resources, transparency of interests, honesty in reporting scientific results (whether favorable, neutral, or negative) and ability to program well-designed studies to offer necessary and accessible information to consumers represent essential and reliable points to characterize any nutrient. Trials lacking adequate scientific design and sufficiently powered samples should immediately be discouraged. Manufacturers in need of positive results to implement products find themselves in conflict with investigators' rules and requirements, especially in cases where the investigators are supposed to substantiate the health claims pertinent to the manufacturers' own products. Because emerging evidence suggests that different genetic backgrounds may drive nutrients toward different metabolic effects in the individual, the functional effects of a given nutrient will become increasingly difficult to assess in small sample studies. Future nutritional intervention trials might need to include specific genetic analyses to adjust for different nutrient susceptibility in the population. This will add a challenge of obtaining permission from Ethics Committees in a field, such as nutrition, in which studies are based on preventive approach rather than a drug treatment approach. Within this context, the limits between virtuous circles, leading to an effective improvement of knowledge and the production of new health enhancing nutrients, and vicious circles, perpetuating biased or unadjusted (in best cases) results with the production of nutrients with limited or no health benefits may hardly be recognized.

Which solution can be proposed? Scientific bodies, such as the ESPGHAN Committee on Nutrition, have already issued indications for adequately planned nutritional study designs (1). Several factors should be considered: the goal for manufacturers to develop new effective products, the goal for investigators to perform valuable research, the opportunity to optimize financial funding in the light of increasingly important cost/benefit ratios, and the final end-point, the health and the well-being of the consumer. A feasible way to achieve these goals would be to study large cohorts of infants directly followed from birth through the pediatric age and beyond. To this end, manufacturers would have to create pools of sponsors (different manufacturers, academic institutions, and private foundations) of cointerested actors. On an administrative level, manufacturers should aim at supporting academic institutions where the research units are situated, rather than directly supporting single researchers and research units. This model is already working within the research projects supported by the European Union and, hopefully, should progressively be applied within all applied nutritional sciences. - Carlo Agostoni

\section{REFERENCE}

1. Aggett P, Agostoni C, Axelsson I, Goulet O, Hernell O, Koletzko B, Lafeber HN, Michaelsen KF, Morley R, Rigo J, Szajewska H, Weaver LT 2003 Core data for nutrition trials in infants: a discussion document-a commentary by the ESPGHAN Committee on Nutrition. J Pediatr Gastroenterol Nutr 36:338-342 